\title{
Patients with infective endocarditis: Five-year observation from a single reference center
}

\author{
Józefa Dąbek ${ }^{1, A, E, F}$, Michał Majewski ${ }^{1}$, A-E , Marta Michalak-Kolarz ${ }^{2, A-D}$, Zbigniew Gąsior ${ }^{11, E, F}$ \\ ${ }^{1}$ Chair and Department of Cardiology, School of Health Sciences, Medical University of Silesia in Katowice, Poland \\ ${ }^{2}$ Chair and Department of Cardiology, Students' Scientific Society, School of Health Sciences, Medical University of Silesia in Katowice, Poland \\ A - research concept and design; B - collection and/or assembly of data; C - data analysis and interpretation; \\ $D$ - writing the article; $E$ - critical revision of the article; $F$ - final approval of the article
}

\section{Józefa Dąbek \\ E-mail:jdabek@sum.edu.pl \\ Funding sources \\ None declared \\ Conflict of interest \\ None declared}

Address for correspondence

Received on February 7, 2016

Reviewed on June 21, 2016

Accepted on August 29, 2016

\begin{abstract}
Background. Infective endocarditis (IE) is a serious and, if untreated, usually fatal disease. Diagnosing IE is often considered to be one of the most difficult medical conditions because of the heterogeneous and ambiguous clinical presentation.
\end{abstract}

Objectives. The purpose of this study was to investigate diagnostic and therapeutic management in a non-selected group of patients with IE.

Material and methods. A total of 45 patients consecutively admitted to the Department of Cardiology, Medical University of Silesia in Katowice (mean age $53.6 \pm 18$ years; 13 females) with IE between 2009 and 2013 were evaluated. Echocardiography, blood cultures and laboratory tests were performed on every patient upon admission. An analysis of the diagnostic and therapeutic management was performed.

Results. Most frequent predisposing factors were: a history of heart valve replacement and/or repair (40\%), dental caries (17.8\%) and bicuspid aortic valve (17.8\%). The majority of patients were admitted from another hospital (91.1\%). Fever (92\%) and symptoms of heart failure (80\%) were the most common manifestations. Abnormalities in ECG occured in $91.2 \%$ of patients. Echocardiography was highly sensitive $(>90 \%)$ in detecting endocardial changes. Staphylococcal etiology was the most common (33.3\%). Surgery procedures were necessary in $62.2 \%$ of patients. Arrhythmias (91.1\%) and acute heart failure (57.8\%) were the most commonly observed complications, although $24.4 \%$ of subjects had neurological disorders. The patients studied were burdened with a number of factors predisposing to IE.

Conclusions. The diagnosis of IE can be a difficult challenge. Particular attention should be paid to the care of oral hygiene and treatment of dental diseases in order to reduce the risk of IE developing.

Key words: etiology, complication, infective endocarditis, treatment

DOI

10.17219/acem/64874

\section{Copyright}

Copyright by Author(s)

This is an article distributed under the terms of the

Creative Commons Attribution Non-Commercial License

(http://creativecommons.org/licenses/by-nc-nd/4.0/) 


\section{Introduction}

Infective endocarditis (IE) is defined as a group of clinical syndromes resulting from an inflammatory process of the endocardium caused by microorganisms, particularly bacteria. Despite major advances in diagnostic and therapeutic procedures, IE still carries poor prognosis and high mortality. Furthermore, neither the incidence nor the mortality has decreased in recent decades. Nevertheless, in developed countries, a typical IE patient has entirely changed over recent years. We can increasingly observe the causes of IE to be associated with health-care contact and such procedures of invasive cardiology or surgery, as the implantation of pacemakers, cardioverter-defibrillators, resynchronization therapy devices, and prosthetic heart valves. More frequently, we have to deal with intravenous drug abuse. On the other hand, patients with a history of rheumatic fever are less common.

The most common location of the inflammatory process is the aortic and mitral valve. In about $10 \%$ of cases, it involves more than 1 valve. The diverse and frequently atypical course of the disease makes the diagnostic process of IE a challenge for clinicians, and requires a multidisciplinary team (endocarditis team) approach. The most common symptom (in almost all patients) is fever. Clinical presentation largely depends on the location of the vegetations. In the case of right-sided endocarditis, beyond the systemic symptoms, pneumonia and congestive heart failure are the predominating complaints. When the process is left-sided, clinical presentation will result from systemic embolism and symptoms of heart failure, often presenting as acute heart failure. Characteristic for IE signs, such as nail hemorrhages, Osler's nodules or Roth's spots, are rather rare in everyday clinical practice.

The first step in the diagnosis of IE is a combination of disease probability based on the patient's history and physical examination supported by the results of additional tests, among which the most important are blood cultures and echocardiography. According to the current guidelines of the European Society of Cardiology, the diagnosis of the disease might be certain or probable, depending on the clinical presentation and results of additional tests, among which molecular diagnostics and nuclear imaging can enhance the diagnosis of IE, particularly in difficult to diagnose cases. Not infrequently, however, blood cultures may be negative, and the etiology remains unknown (BCNIE, blood culture negative infective endocarditis).

Since IE is a relatively rare disease, we do not have data from large, randomized clinical trials. IE could result in a number of complications, such as endocardium and valvular damage, systemic embolism or harmful immune response of the body, and in many cases, apart from optimal pharmacotherapy, also require surgical procedures. If left untreated, endocarditis, which is in fact a generalized infection, usually leads to death. This article provides an overview of an unselected group of patients with IE, and allows insight into the diagnostic and therapeutic procedures of that group of patients in daily clinical practice.

\section{Material and methods}

The aim of the study was to evaluate patients with IE hospitalized in the Department of Cardiology, Medical University of Silesia in Katowice, Poland.

Consecutive patients admitted with IE to the Department of Cardiology between 2009 and 2013 were evaluated. The study group involved 45 patients, aged from 18 to

Table 1. Baseline characteristics of the study group (mean age $53.6 \pm 18$ years)

\begin{tabular}{|c|c|c|}
\hline Parameter & $\mathrm{n}$ & $\%$ \\
\hline All studied group & 45 & 100 \\
\hline \multirow{2}{*}{ Sex } & 13 & 29 \\
\hline & 32 & 71 \\
\hline \multicolumn{3}{|c|}{ Comorbidities } \\
\hline Arterial hypertension & 16 & 35.6 \\
\hline Diabetes & 6 & 13.3 \\
\hline Atrial fibrillation & 6 & 13.3 \\
\hline Ischaemic heart disease & 4 & 8.9 \\
\hline History of acute coronary syndromes & 1 & 2.2 \\
\hline Peripheral artery disease & 1 & 2.2 \\
\hline \multicolumn{3}{|c|}{ Referral place } \\
\hline Department of Internal Medicine & 36 & 79.8 \\
\hline Department of Cardiac Surgery & 4 & 9 \\
\hline GP office & 2 & 4.5 \\
\hline House (ambulance) & 2 & 4.5 \\
\hline Department of Neurology & 1 & 2.2 \\
\hline \multicolumn{3}{|c|}{ Initial diagnosis } \\
\hline Infective endocarditis & 38 & 84 \\
\hline Cardiac arrhythmias & 3 & 7 \\
\hline Acute coronary syndrome & 2 & 4.5 \\
\hline Heart failure aggravation & 2 & 4.5 \\
\hline \multicolumn{3}{|c|}{ Laboratory findings (mean \pm standard deviation) } \\
\hline $\begin{array}{l}\text { Erythrocyte sedimentation rate }[\mathrm{mm} / \mathrm{h}] \\
(\mathrm{n}<15)\end{array}$ & \multicolumn{2}{|c|}{$60 \pm 36$} \\
\hline Leukocytes $\left[\times 10^{3} / \mu \mathrm{L}\right](\mathrm{n}: 4-10)$ & \multicolumn{2}{|c|}{$9.67 \pm 6.38$} \\
\hline Erythrocytes $\left[\times 10^{6} / \mu \mathrm{L}\right](\mathrm{n}: 4.2-5.4)$ & \multicolumn{2}{|c|}{$3.82 \pm 0.97$} \\
\hline Hemoglobin [g/dL] (n: 12-18) & \multicolumn{2}{|c|}{$11.1 \pm 2.3$} \\
\hline C-reactive protein [mg/L] (n: 0.08-3.1) & & \\
\hline Total cholesterol [mg/dL] $(\mathrm{n}<200)$ & & \\
\hline LDL cholesterol [mg/dL] $(\mathrm{n}<115)$ & & \\
\hline HDL cholesterol [mg/dL] $(n>45)$ & & \\
\hline Triglycerides [mg/dL] $(\mathrm{n}<150)$ & & \\
\hline Glucose $[\mathrm{mg} / \mathrm{dL}](\mathrm{n}<100)$ & & \\
\hline Creatinine $[\mathrm{mg} / \mathrm{dL}](\mathrm{n}: 0.6-1.3)$ & & 35 \\
\hline
\end{tabular}

$n-$ number 
78 years. The mean age was 53.6 years, and $71.1 \%(n=32)$ of patients were men.

A detailed analysis of all patients was performed including medical history, physical examination and laboratory tests: complete blood count, concentration of C-reactive protein, erythrocyte sedimentation rate (ESR), glucose, creatinine and lipids (total, LDL and HDL cholesterol, triglycerides); bacteriological: blood cultures for aerobic and anaerobic bacteria; imaging: transthoracic echocardiography (TTE), transoesophageal echocardiography (TEE), coronary angiography and other: electrocardiogram (ECG) and blood pressure measurements. Blood cultures were taken 3 times in half-hour intervals.

Continuous data was presented as mean \pm standard deviation, and categorical data was presented as frequencies and percentages.

\section{Results}

\section{Characteristics of the study group}

Baseline characteristics of the patients studied are presented in Table 1.
The study group involved $71.1 \%(\mathrm{n}=32)$ men, wherein 12 individuals were under 40 years old, 15 were between 40 and 60 years, and 18 patients were more than 60 years old. Arterial hypertension was the most common comorbidity. The majority of patients in the period preceding hospitalization in the Cardiology Unit were diagnosed in other hospital wards, most commonly at internal medicine units. IE was the most common initial diagnosis and cause of referral to the reference Department of Cardiology.

The prevalence of identified predisposing factors is shown in Fig. 1.

The individuals studied were burdened with multiple risk factors for IE, particularly a history of heart valve operations (repair and/or replacement). Also, a high prevalence of bicuspid aortic valve and advanced dental caries were revealed.

\section{Clinical presentation}

The type of signs and symptoms and their incidence in the group of patients with IE studied is shown in Fig. 2.

The most frequent findings in the evaluated group of patients were high body temperature $(91.1 \%$ of subjects had fever) and symptoms of heart failure ( $80 \%$ of patients),

Fig. 1. Factors predisposing to infective endocarditis in the study group

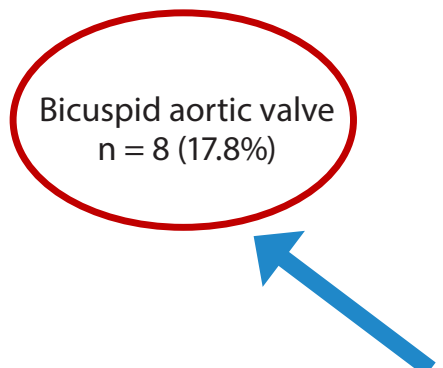

Prior infective $\mathrm{n}=5(11.1 \%)$

Congenital heart defect $\mathrm{n}=1(2.2 \%)$ The presence of predisposing factors for the development of infective endocarditis (IE) in the study group

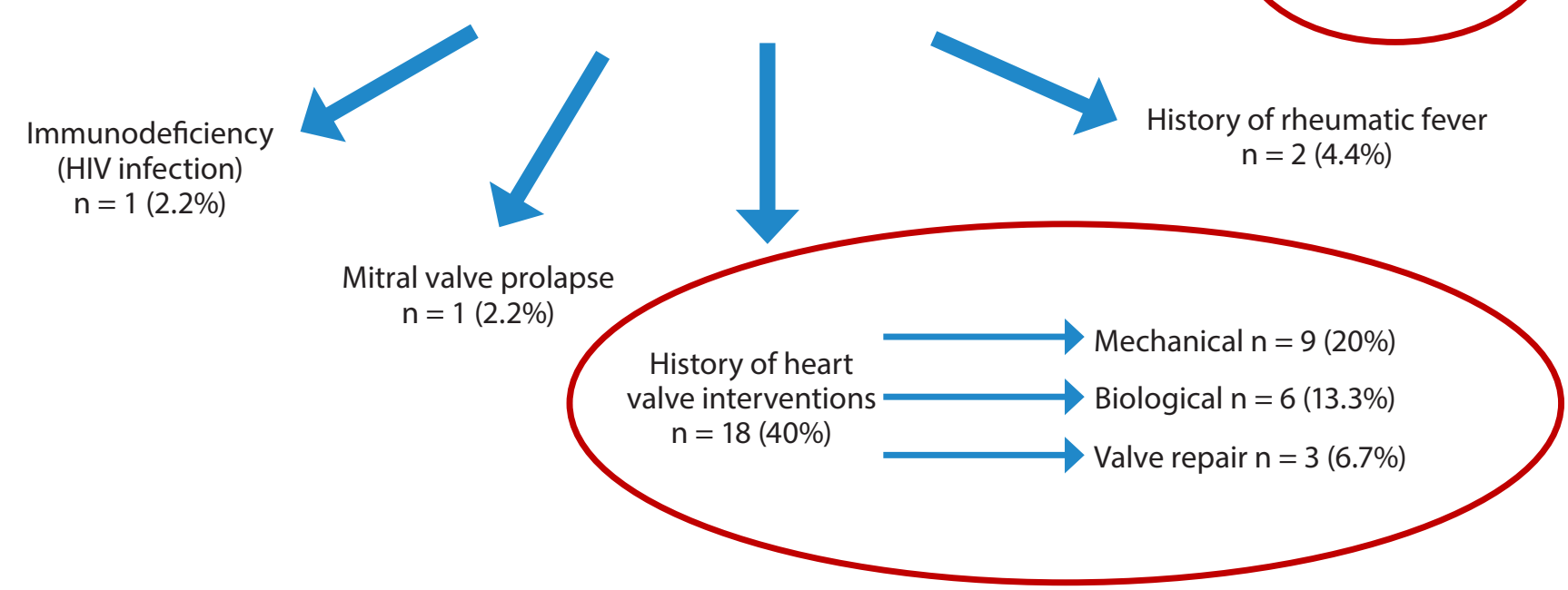


including acute heart failure resulting from complications of IE in almost $20 \%$ of the patients studied. Likewise, a high incidence of central nervous system complications was noticed.

Fig. 2. Characteristics of the group of patients with infective endocarditis studied according to the clinical symptoms

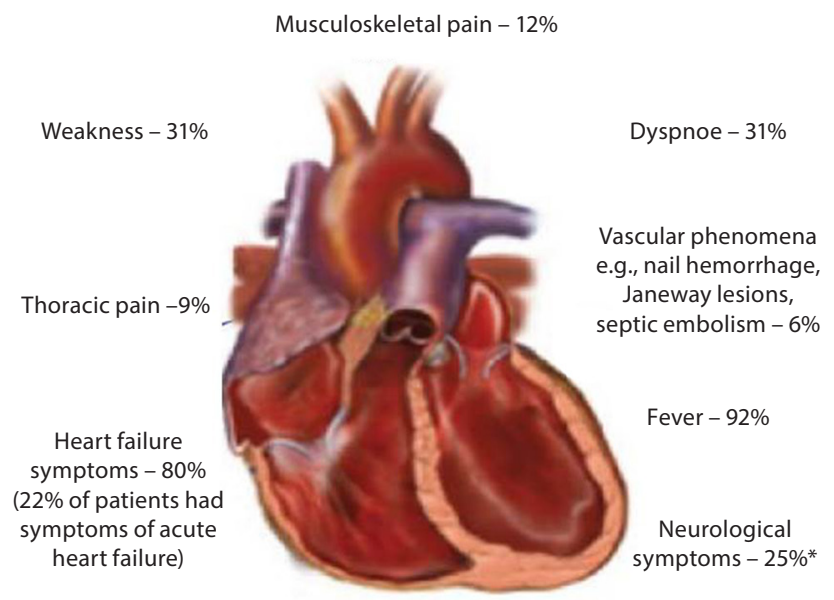

* - headache, confusion, loss of consciousness, weakness, impaired vision.

\section{Investigations}

\section{Laboratory tests}

Elevated levels of C-reactive protein and ESR were observed in all patients with endocarditis. Increased leukocytosis and anaemia were revealed in a majority of patients, in which 4 patients $(8.8 \%)$ required blood transfusion.

\section{2-lead ECG}

Only a few patients with endocarditis had normal ECG records ( $\mathrm{n}=4 ; 8.8 \%$ ). The most frequently ascertained cardiac arrhythmias were atrial fibrillation (20\% of patients) and supra- and ventricular extrasystoles (15.6\% of patients). When conduction disturbances are involved, the most frequent was right bundle branch block $(13.3 \%$ of patients).

\section{Microbial tests (blood cultures)}

The results of the microbiological investigations' attempts to find the etiology of IE and the type of antibiotics used during treatment are shown in Fig. 3.

Fig. 3. The results of blood cultures and cultured microorganisms in the group of patients with infective endocarditis studied. Frequency of use of antibiotics in regard to the results of antibiogram

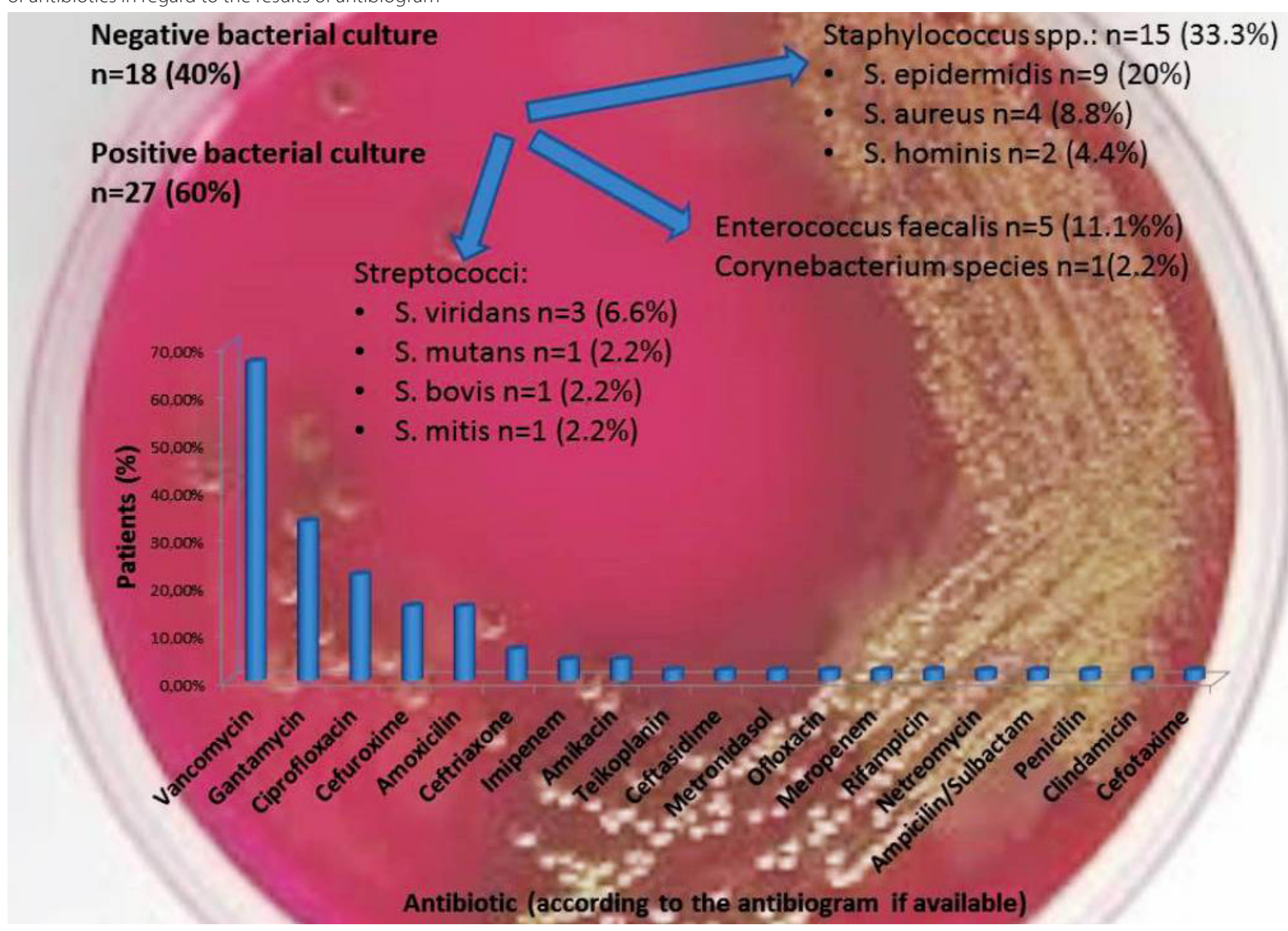




\section{Echocardiography}

Standard transthoracic echocardiography (TTE) was performed in every patient, and additionally, in the case of poor quality of TTE or when there were other indications (such as a prosthetic valve), transoesophageal echocardiography (TEE) was also performed. The results obtained from the echocardiography are presented in Table 2.

Only 4 (8.8\%) patients revealed no echocardiographic changes, and among them half had already been treated with antibiotics. The most common location of bacterial vegetations was the aortic and mitral valve. The vast majority of changes were located in the left part of the heart, and in 1 patient with right-sided endocarditis it was associated with pacemaker lead implantation into the right ventricle (CDRIE, cardiac device-related infective endocarditis). Mitral valve insufficiency was usually caused by the presence of abscess or vegetation that made valve leaflets impossible to cohere. However, in the case of acute mitral insufficiency, the perforation of the valve

Table 2. Echocardiography results

\begin{tabular}{|c|c|c|c|c|}
\hline Parameter & \multicolumn{2}{|c|}{$\overline{\mathrm{x}}$} & \multicolumn{2}{|c|}{ $\pm S D$} \\
\hline LVEF [\%] (n: 55-70\%) & \multicolumn{2}{|c|}{$55 \%$} & \multicolumn{2}{|c|}{$9 \%$} \\
\hline IVS [mm] (n: 6-12 mm) & \multicolumn{2}{|c|}{$11.6 \mathrm{~mm}$} & \multicolumn{2}{|c|}{$2.2 \mathrm{~mm}$} \\
\hline PW [mm] (n: 6-12 mm) & \multicolumn{2}{|c|}{$10.6 \mathrm{~mm}$} & \multicolumn{2}{|c|}{$1.6 \mathrm{~mm}$} \\
\hline Ascending aorta [mm] (n: 20-38 mm) & \multicolumn{2}{|c|}{$32 \mathrm{~mm}$} & \multicolumn{2}{|c|}{$5.6 \mathrm{~mm}$} \\
\hline \multicolumn{5}{|c|}{ Vegetations [ $n=41$ (91.2\% of studied group)] } \\
\hline Localization of vegetations & \multicolumn{2}{|c|}{$\mathrm{n}$} & \multicolumn{2}{|c|}{$\%$} \\
\hline Aortic valve & \multicolumn{2}{|c|}{26} & \multicolumn{2}{|c|}{58} \\
\hline Mitral valve & \multicolumn{2}{|c|}{15} & \multicolumn{2}{|c|}{13.3} \\
\hline Wall endocardium & \multicolumn{2}{|c|}{2} & \multicolumn{2}{|c|}{4.4} \\
\hline Intracardiac devices & \multicolumn{2}{|c|}{1} & \multicolumn{2}{|c|}{2.2} \\
\hline No vegetations & \multicolumn{2}{|c|}{4} & \multicolumn{2}{|c|}{8.8} \\
\hline \multicolumn{5}{|c|}{ Pathology of heart valves } \\
\hline Valve insufficiency & \multicolumn{2}{|c|}{ Mitral valve, n (\%) } & \multicolumn{2}{|c|}{ Aortic valve, $\mathrm{n}(\%)$} \\
\hline Mild & 7 & 15.6 & 0 & 0 \\
\hline Moderate & 11 & 24.4 & 2 & 4.4 \\
\hline Severe & 9 & 20 & 11 & 24.4 \\
\hline Leaflet perforation & 5 & 11.1 & 3 & 3.7 \\
\hline Ruptured chordae tendineae & 1 & 2.2 & 0 & 0 \\
\hline \multicolumn{5}{|c|}{ Other complications } \\
\hline Abscesses & $\begin{array}{c}5 \text { (all } \\
\text { perforated) }\end{array}$ & 11.1 & 6 & 13.3 \\
\hline Pseudoaneurysms & 0 & 0 & 1 & 2.2 \\
\hline Intracardiac fistulas & 1 & 2.2 & 2 & 4.4 \\
\hline \multicolumn{5}{|c|}{ Others } \\
\hline Vegetations in RV & \multicolumn{4}{|c|}{$n=1(2.2 \%)$ (lead of the pacemaker) } \\
\hline Pericardial effusion & \multicolumn{4}{|c|}{$n=7(15.6 \%)$} \\
\hline
\end{tabular}

$\bar{x}$ - arithmetic mean; SD - standard deviation; LVEF - left ventricle ejection fraction; IVS interventricular septum; PW - posterior wall of the left ventricle; $n$ - number. leaflet was ascertained. All cases of mitral valve abscesses had perforated while only one-third of the aortic valve abscesses did.

\section{Treatment}

Optimal medical therapy was administered in every patient studied. Surgical treatment was required in $62.2 \%$ of induviduals, both as immediate (within $24 \mathrm{~h}$ ) or urgent (in a few days) operations, mainly due to heart failure caused directly by an inflammatory process of the valves and its damage. The treatment management of patients with IE is presented in Fig. 4.

The majority of patients also had typical heart failure treatment administered, including beta-blockers ( $\mathrm{n}=34$; $75.6 \%)$, mineralocorticoid receptor antagonists $(n=25$; $55.6 \%$ ), diuretics ( $\mathrm{n}=21 ; 46.7 \%$ ), angiotensin converting enzyme inhibitors or angiotensin receptor blockers $(n=22$; 48.9\%). Moreover, a substantial part of the group studied was treated with vitamin $\mathrm{K}$ antagonists $(\mathrm{n}=5 ; 11.1 \%)$ and low molecular weight heparins $(n=22$; $48.9 \%)$. Blood transfusions were required in 4 patients due to severe anemia (8.8\%).

\section{Complications of IE}

The type and incidence of complications in the group of patients with infective endocarditis studied are presented in Table 3.

An example of heart valve destruction by the inflammatory process is shown in Fig. 5.

\section{Discussion}

IE is hematogenous, systemic infection involving heart structures, and less frequently large blood vessels of the thoracic cavity or foreign materials implanted into heart cavities. IE has a diverse etiology and clinical manifestation and often requires different procedures in individual patients. In order to make diagnostic and therapeutic decisions easier, there are guidelines on IE. We currently rely on the guidelines prepared by the European Society of Cardiology (ESC) in 2015. ${ }^{1}$

The incidence of IE is relatively low and ranges from 3 to 10 episodes per 100,000 person per year., ${ }^{2,3}$ There are 3000 new cases of IE diagnosed annually in Poland. Low incidence makes it difficult to carry out randomized 
clinical trials and meta-analyses. This results in problems with the elaboration of guidelines and providing it to clinicians.

Despite major advances in diagnostic and therapeutic procedures, IE still carries a poor prognosis and high mortality. Furthermore, neither the incidence nor the mortality has decreased in recent decades. On the other hand,

Table 3. IE complications observed during hospitalization

\begin{tabular}{|l|c|c|}
\multicolumn{1}{|c|}{ The study group of patients with IE } & $\mathrm{n}=45$ & $100 \%$ \\
\hline \multicolumn{1}{|c|}{ Complication } & $\mathrm{n}$ & $\%$ \\
\hline Cardiac arrhytmias and conducting abnormalities & 41 & 91 \\
\hline Valve damage (requiring surgery) & 26 & 58 \\
\hline Cerebral stroke & 8 & 17.8 \\
\hline Cardiogenic shock & 7 & 15.6 \\
\hline Acute left ventricular failure (pulmonary edema) & 5 & 11.1 \\
\hline Septic shock & 5 & 11.1 \\
\hline Acute kidney injury & 4 & 8.8 \\
\hline Pathological intracardiac connections (fistulas) & 3 & 6.7 \\
\hline Sudden cardiac arrest & 2 & 4.4 \\
\hline Transient ischaemic attack of CNS & 1 & 2.2 \\
\hline Multiple cerebral abscesses & 1 & 2.2 \\
\hline
\end{tabular}

IE - infective endocarditis; $\mathrm{n}$ - number; CNS - central nervous system. there have been some changes in the etiology of IE and the factors predisposing for its development. ${ }^{4}$ This situation is associated with an increasing number of medical procedures in cardiovascular diseases, such as implantation of intracardiac devices (pacemakers, ICD, devices for resynchronization therapy) and prosthetic valves (in the study group, $40 \%$ of patients had prior valve replacement), as well as an increasing number of patients undergoing invasive procedures for non-cardiac reasons such as hemodialysis. ${ }^{5-7}$

Changes in antibiotic use have undoubtedly had a huge impact on the alteration in etiology. ${ }^{8,9}$ Staphylococcus, especially $S$. epidermidis, was the most frequent etiological factor in the group studied. A metaanalysis concerning etiological changes over the last half century delivered interesting data in this area, indicating an outstanding increase in staphylococcal infections (formerly dominated by streptococci). Although an increased frequency of staphylococcal endocarditis is observed in most developed countries, this is particularly evident in the United States, and most probably results from an increase in intravenous drug abuse. ${ }^{10}$ Even so, it seems that in Europe it is rather connected with an increasing number of invasive health-care-associated procedures (mainly in invasive cardiology and electrocardiology).

Fig. 4. The type of in-hospital treatment of patients with infective endocarditis with the urgency of surgery performed

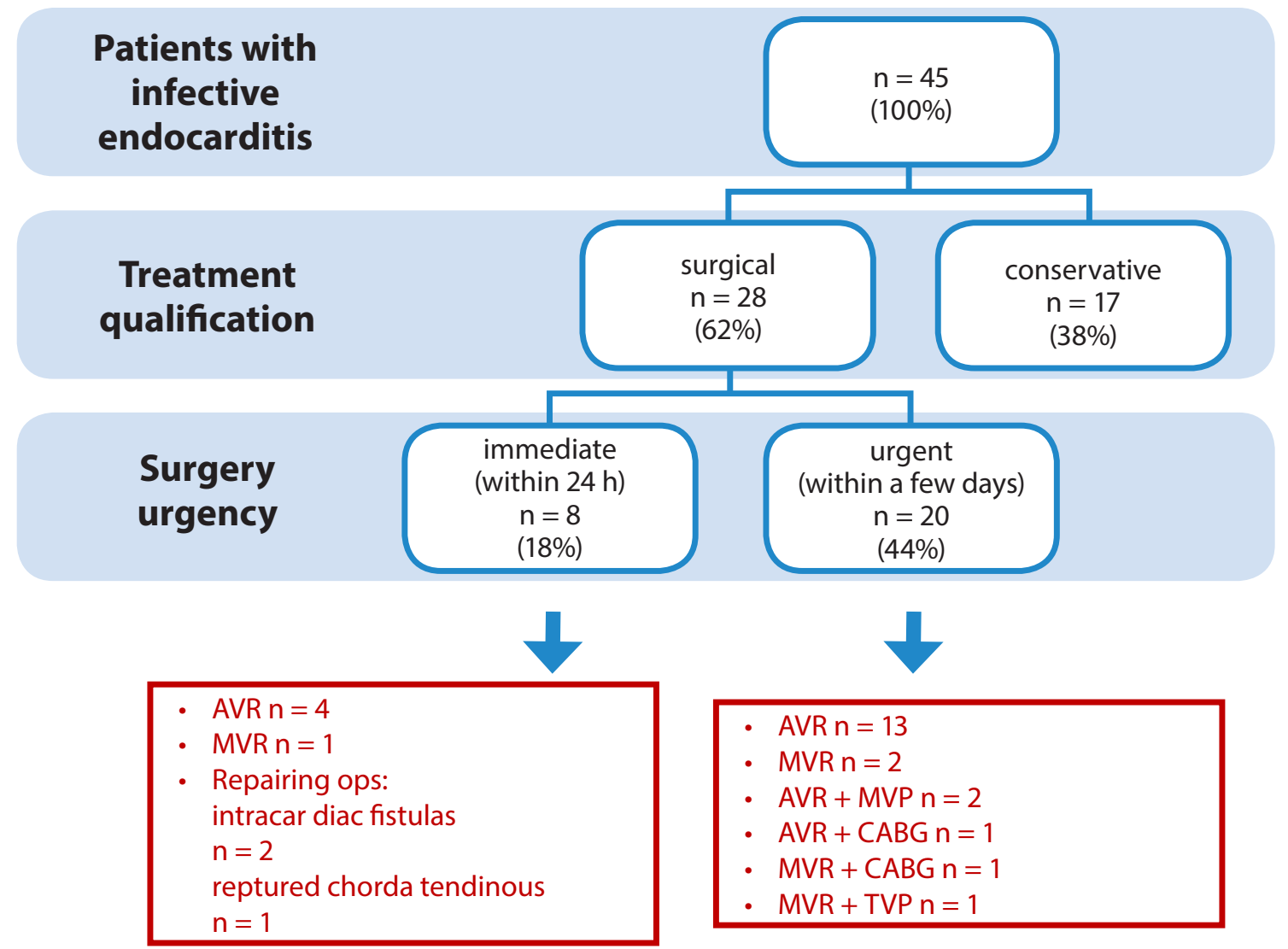

$\mathrm{n}$ - number; AVR - aortic valve replacement; MVR - mitral valve replacement; MVP - mitral valve repair; CABG - coronary artery by-pass grafting; TVP - tricuspid valve repair. 
The same meta-analysis has also shown an increased incidence of enterococcal endocarditis. Moreover, patients affected with this etiology were more often in worse general health condition, older, diabetic, had valve impairment, were dialyzed or with immunosuppression. ${ }^{11}$ Similar conclusions can be drawn from our study - the third most common etiology of IE (enterococci) was particularly evident in severely ill patients. This is particularly important in regard to increasing antibiotic resistance. In developing countries, the classic risk factors predisposing to IE (especially rheumatic heart disease) are present, and most frequently streptococcal etiology is still observed. ${ }^{10}$ Over the last decades, a typical patient with IE, a young person with a history of rheumatic fever, has changed, and nowadays it is most often an older patient, burdened with a number of comorbidities, after prior cardiac surgery or cardiology intervention. In the group studied, men predominate, which is consistent with most observations, although some authors did not demonstrate such a relationship. ${ }^{3}$

IE is not homogeneous disease but rather a set of clinical conditions. Current European guidelines distinguish the following types of endocarditis, according to the site of infection and the presence or absence of intracardiac prosthetic material - left-sided native valve IE (NVE), left-sided prosthetic valve IE (PVE), right-sided IE, and device-related IE (the latter including IE developing on pacemaker or defibrillator wires with or without associated valve involvement, CDRIE). With regard to acquisition, the following situations can be distinguished: community-acquired IE, health care-associated IE (nosocomial and non-nosocomial) and IE in intravenous drug abusers. ${ }^{1}$ It is most frequently localized in a heart valve but can also be located in the wall endocardium of the atria or ventricles, the blood vessels and foreign material located in the heart (valve, electrode). In the group of patients with IE studied, the most common location was in a replaced or degenerated aortic valve. In 1 patient, a lesion was located in the right heart and involved a pacemaker wire. Right-sided endocarditis is a rare condition affecting up to $5 \%$ of all cases. ${ }^{1}$ The cause is usually related to intravenous drug abuse, although the number of rightsided infections also increases in the group of patients with implanted electrodes and catheters, as was presented in our study.

The clinical presentation of IE, as shown in this study, can be very diverse, and is dependent on variety of factors such as: the location of the inflammatory process,

Fig. 5. Echocardiographic image of infective endocarditis complication - perforation of aortic valve in 1 of the patients studied

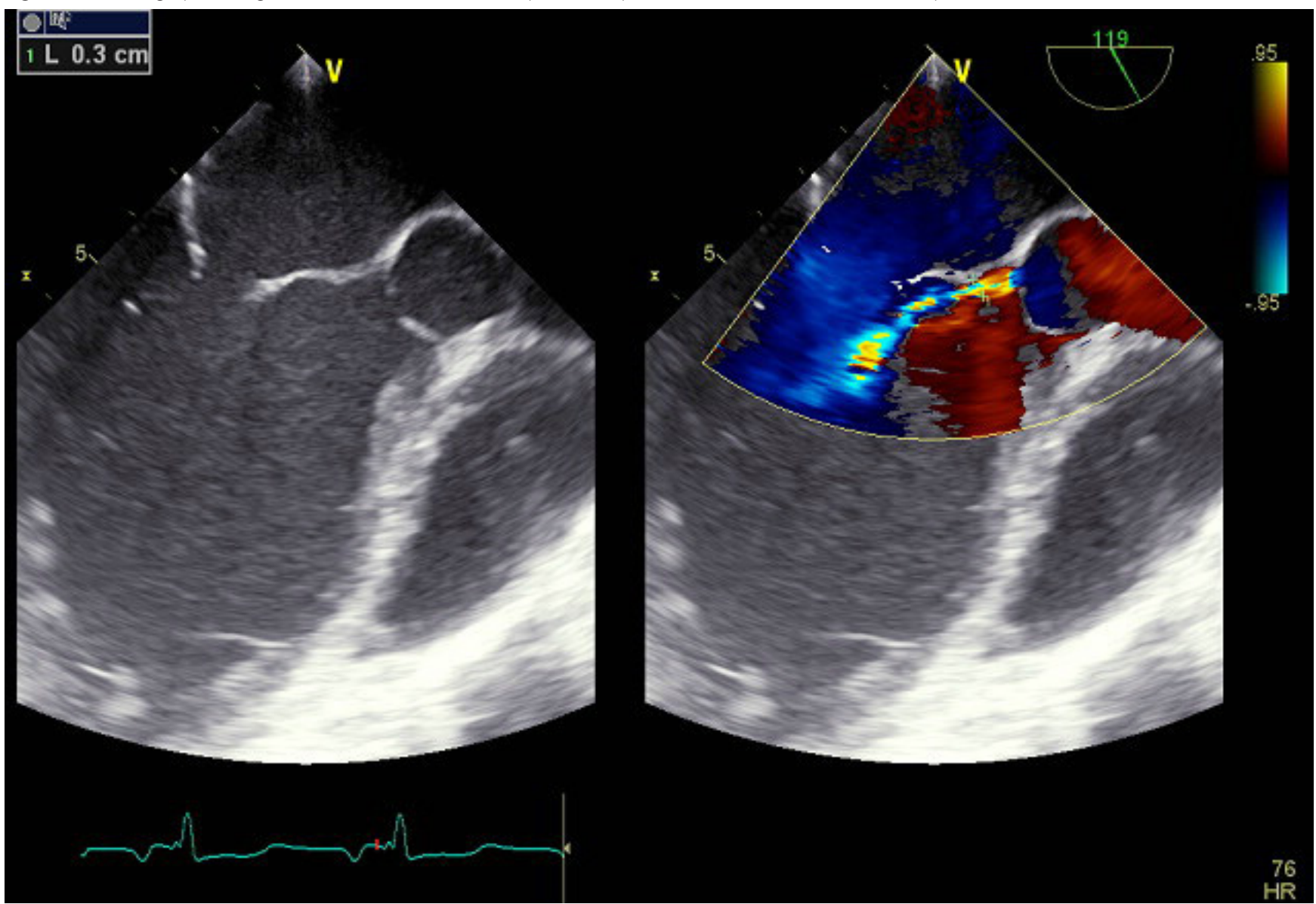

A - 2D imaging, perforation place is indicated with yellow arrow; B - color Doppler, perforation is seen as multicolor blood leak through the valve leaflets (green arrow). Source: institutional records. 
the age of the patient, immunological disorders or prior use of antibiotics. More and more often, the manifestation of IE is atypical, particularly in older individuals and those with immunosuppression. ${ }^{12}$

Despite a patient's medical history and physical examination, echocardiographic and microbiological tests play an essential role in the diagnosis of IE. TTE is a first-choice test when IE is suspected. The majority should also have TEE performed, particularly if the image of the TTE is of poor quality. Echocardiography is not only necessary to confirm the diagnosis of IE but also plays a key role in making therapeutic decisions, predicting disease course and confirming complications, but is also used for periodic checkups of the patient in the long term. Only 4 patients showed no characteristics for IE lesions in the TTE, of which half (2 persons) had already been administered antibiotics. The sensitivity of echocardiography in IE diagnosis is acceptable and ranges from 40 to $63 \%$ for TTE and from 90 to $100 \%$ for TEE. ${ }^{13}$ However, many situations might hinder an echocardiographic diagnosis of IE. Notwithstanding, one should still notice increasing opportunities due to the development of echocardiographic techniques. Modified Duke University Criteria allow for the diagnosis of the majority of cases of IE (80\%). ESC 2015 guidelines on IE emphasize the use of new diagnostic approaches, both molecular and imaging techniques such as computed tomography (CT), positron emission tomography (PET), single photon emission computed tomography (SPECT) with radiolabeled leukocytes, and cardiac magnetic resonance (CMR) in particular, diagnostically difficult cases (BCNIE, PVE, CDRIE). Abnormal activity around the site of prosthetic valve implantation detected by ${ }^{18} \mathrm{~F}-\mathrm{FDG}$ PET/CT or radiolabelled leukocytes SPECT/CT or definite paravalvular lesions detected by CT are major criteria while vascular phenomena (even clinically silent, seen only in imaging modalities) is a minor ESC IE criterion. ${ }^{18} \mathrm{~F}-\mathrm{FDG} \mathrm{PET} / \mathrm{CT}$ increased the sensitivity of Duke Criteria from 70 to 97\% in the case of PVE. However, it allows early diagnosis and complication assessment such as emboli and metastatic infections as well as possibly revealing other sources of infection (whole body PET/CT). It could be non-specific in some situations however (inflammation, early postoperative period).

Blood cultures play a key role in determining etiology and its susceptibility to antibiotics. It is worth remembering that bacteremia in IE is almost constant, which has clinical implications (therefore, do not delay blood sampling to coincide with a peak of fever). An etiological factor mustn't be specified based on a result of a single blood culture (3 blood samples in 30-min intervals should be taken prior the antibiotics). The most commonly-used criteria for diagnosing IE are modified Duke University Criteria characterized by a relatively high sensitivity and specificity, which have recently been supported by new molecular and nuclear imaging diagnostic approaches in the ESC 2015 document. They are based upon clinical, echocardiographic and microbiological findings. Even so, they do not allow omitting clinical judgment, which is the most important criterion. An assessment of the prognosis should follow a diagnosis by taking the following into consideration: patient characteristics, complication occurrence, type of microorganism and echocardiography results that make it possible to identify emerging high-risk patients requiring urgent surgical treatment and precise monitoring in order to improve outcomes. About $30 \%$ of subjects with strong clinical suspicion of IE are blood culture negative (BCNIE). Molecular techniques are useful in both the detection and identification of the causal organisms of IE. ESC 2015 guidelines propose a serological diagnosis followed by PCR approaches in the case of BCNIE. New PCR methods such as PCR electrospray ionization-mass spectrometry (PCR-ESI-MS) are available. Matrix-assisted laser desorption ionization time of flight mass spectrometry (MALDI-TOF MS) is an example of a non-PCR molecular approach, which is more cost-effective and allows for identification of causal agents directly from blood cultures (no need of gene amplification).

Proper treatment of IE always includes pharmacotherapy and in many situations surgical intervention. A fundamental principle is antimicrobial therapy and frequently treatment of complications resulting from the inflammatory process, particularly heart failure. The aim of antibiotic therapy is microbial eradication.

Duration of treatment depends on the pathogen type and location of the endocarditis. The native valve endocarditis (NVE) usually needs to be treated for 2-6 weeks, while IE of a prosthetic valve should last longer (at least 6 weeks). Because the treatment of IE should be started promptly (before the results of blood cultures), usually empirical antibiotic therapy is administered as well as in cases of non-identification of the microorganism. After receiving the results of the antibiogram, drugs should be adjusted to its results. In the study group, $62.2 \%$ of patients were operated on during hospitalization. It is said that about $50 \%$ of patients need surgical procedures because of endocarditis. ${ }^{2,14}$ Indications for immediate or urgent surgery results from the presence of severe heart failure, uncontrollable infection and thromboembolic events. The most common reason for surgery in active endocarditis is the emergence of acute heart failure.

IE, if left untreated, is a lethal condition. Complications of IE, including death, are quite common, even if adequate therapy is administered. In the group of patients studied, the most common complications were different forms of heart failure. A high incidence of neurological disorders, especially cerebral stroke, were observed as well. Neurological complications occur in approx. 1/3 of patients with endocarditis. ${ }^{15,16}$ Likewise, in the study group, the most common were 
cerebral stroke and transient ischemic attack of the central nervous system (TIA) or on the other hand they could be asymptomatic and caused by embolic material detached from vegetations. A systemic embolism makes prognosis poorer, and the most important prevention method in embolic complications is an early diagnosis of IE, followed by appropriate antibiotic therapy. ${ }^{17} \mathrm{De}-$ spite these neurological adverse effects, most patients are still candidates for surgical treatment that could improve their outcomes. ${ }^{16}$

In recent years, a number of guidelines for IE have been published, which have completely changed the approach to antibiotic prophylaxis. ${ }^{18}$ The guidelines of the American Heart Association from 2007 were the first that advised a complete change in the current management, reducing significantly the number of candidates to people in whom IE is associated with a very poor prognosis (implanted valve prostheses, prior endocarditis, some congenital cyanotic heart defects, heart transplantation with impaired function of the valves). When deciding on the use of antibiotic prophylaxis, we should also consider the type of medical interventions performed and additional circumstances. The 2008 NICE (National Institute for Health and Care Excellence) and 2015 ESC guidelines on antibiotic prophylaxis reduced the number of patients in which such prophylaxis could be beneficial. ${ }^{1,19}$ In these documents, it is emphasized that there is insufficient evidence on the effectiveness of antibiotic prophylaxis for IE, and that the common use of antibiotics can result in more side effects than benefits. Further, more attention must be paid to oral hygiene as one of the most successful types of IE prophylaxis.

\section{Conclusions}

In the group of patients with IE studied, there was a more frequent occurrence of predisposing factors, especially a history of valve replacement and/or repair interventions, the presence of bicuspid aortic valve and advanced tooth decay. Diagnosis of IE still remains a challenge. In a majority, ECG abnormalities, although not specific for IE, were observed, and echocardiography confirmed its usefulness in the visualization of characteristic for IE lesions. IE in the patients studied was associated with a high rate of complications such as structural damage of the heart and neurological events. Most of the patients, in addition to optimal medical therapy, required surgery. The presence of risk factors for developing endocarditis, particularly the occurrence of advanced dental caries, requires extensive health education from an early age.

\section{References}

1. Habib G, Lancellotti P, Antunes MJ, et al. 2015 ESC Guidelines for the management of infective endocarditis. Eur Heart J. 2015;36(44):3075-3128. doi: 10.1093/eurheartj/ehv319.

2. Hoen B, Alla F, Selton-Suty C, et al. Association pour l'Etude et la Prévention de I'Endocardite Infectieuse (AEPEI) Study Group. Changing profile of infective endocarditis: Results of a 1-year survey in France. JAMA. 2002;288:75-81.

3. Hogevik H, Olaison L, Andersson R, Lindberg J, Alestig K. Epidemiologic aspects of infective endocarditis in an urban population. A 5-year prospective study. Medicine (Baltimore). 1995;74:324-339.

4. Moreillon P, Que YA. Infective endocarditis. Lancet. 2004;363:139-149.

5. Cabell CH, Heidenreich PA, Chu VH, Moore CM, Stryjewski ME, Corey GR, Fowler VG Jr. Increasing rates of cardiac device infections among Medicare beneficiaries: 1990-1999. Am Heart J. 2004;147:582-586.

6. Darouiche RO. Treatment of infections associated with surgical implants. N Engl J Med. 2004;350(14):1422-1429.

7. McCarthy JT, Steckelberg JM. Infective endocarditis in patients receiving long-term hemodialysis. Mayo Clin Proc. 2000;75:1008-1014.

8. Wisplinghoff H, Bischoff T, Tallent SM, Seifert H, Wenzel RP, Edmond MB. Nosocomial bloodstream infections in US hospitals: Analysis of 24179 cases from a prospective nationwide surveillance study. Clin Infect Dis. 2004;39:309-317.

9. Fluit AC, Jones ME, Schmitz FJ, Acar J, Gupta R, Verhoef J. Antimicrobial susceptibility and frequency of occurrence of clinical blood isolates in Europe from the SENTRY antimicrobial surveillance program, 1997 and 1998. Clin Infect Dis. 2000;30:454-460.

10. Slipczuk L, Codolosa JN, Davila CD, et al. Infective endocarditis epidemiology over five decades: A systematic review. PLoS One. 2013;9;8:e82665. doi: 10.1371/journal.pone. 0082665.

11. Munita JM, Arias CA, Murray BE. Enterococcal endocarditis: Can we win the war? Curr Infect Dis Rep. 2012;14:339-349.

12. Perez de Isla L, Zamorano J, Lennie V, Vazquez J, Ribera JM, Macaya C. Negative blood culture infective endocarditis in the elderly: Longterm follow-up. Gerontology. 2007;53:245-249.

13. Evangelista A, Gonzalez-Alujas MT. Echocardiography in infective endocarditis. Heart. 2004;90:614-617.

14. Bertagna F, Bisleri G, Motta F, et al. Possible role of F18-FDG-PET/CT in the diagnosis of endocarditis: Preliminary evidence from a review of the literature. Int J Cardiovasc Imaging. 2012;28(6):1417-1425.

15. Wang A, Athan E, Pappas PA, et al. International collaboration on endocarditis-prospective cohort study investigators. Contemporary clinical profile and outcome of prosthetic valve endocarditis. JAMA. 2007;297(12):1354-1361.

16. Thuny F, Avierinos JF, Tribouilloy C, et al. Impact of cerebrovascular complications on mortality and neurologic outcome during infective endocarditis: A prospective multicentre study. Eur Heart J. 2007;28:1155-1161.

17. Anderson DJ, Goldstein LB, Wilkinson WE, et al. Stroke location, characterization, severity and outcome in mitral vs aortic valve endocarditis. Neurology. 2003;61:1341-1346.

18. Heiro M, Nikoskelainen J, Engblom E, Kotilainen E, Marttila R, Kotilainen P. Neurologic manifestations of infective endocarditis: A 17-year experience in a teaching hospital in Finland. Arch Intern Med. 2000;160:2781-2787.

19. Wilson W, Taubert KA, Gewitz M, et al. Prevention of infective endocarditis: Guidelines from the American Heart Association: A guideline from the American Heart Association Rheumatic Fever, Endocarditis, and Kawasaki Disease Committee, Council on Cardiovascular Disease in the Young, and the Council on Clinical Cardiology, Council on Cardiovascular Surgery and Anesthesia, and the Quality of Care and Outcomes Research Interdisciplinary Working Group. Circulation. 2007;116:1736-1754.

20. NICE Short Clinical Guidelines Technical Team. Prophylaxis against infective endocarditis: Antimicrobial prophylaxis against infective endocarditis in adults and children undergoing interventional procedures. London: National Institute for Health and Clinical Excellence; 2008. 